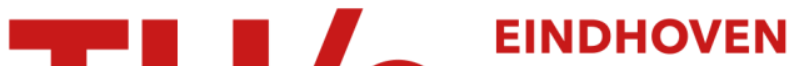 UNIVERSITY OF TECHNOLOGY
}

\section{Rotation of a strongly magnetized hydrogen plasma column determined from an asymmetric Balmer-beta spectral line with two radiating distributions}

\author{
Citation for published version (APA): \\ Shumack, A. E., Veremiyenko, V. P., Schram, D. C., Blank, de, H. J., Goedheer, W. J., Meiden, van der, H. J., \\ Vijvers, W. A. J., Westerhout, J., Lopes Cardozo, N. J., \& Rooij, van, G. J. (2008). Rotation of a strongly \\ magnetized hydrogen plasma column determined from an asymmetric Balmer-beta spectral line with two \\ radiating distributions. Physical Review E - Statistical, Nonlinear, and Soft Matter Physics, 78(4), [046405]. \\ https://doi.org/10.1103/PhysRevE.78.046405
}

DOI:

10.1103/PhysRevE.78.046405

Document status and date:

Published: 01/01/2008

\section{Document Version:}

Publisher's PDF, also known as Version of Record (includes final page, issue and volume numbers)

\section{Please check the document version of this publication:}

- A submitted manuscript is the version of the article upon submission and before peer-review. There can be important differences between the submitted version and the official published version of record. People interested in the research are advised to contact the author for the final version of the publication, or visit the $\mathrm{DOI}$ to the publisher's website.

- The final author version and the galley proof are versions of the publication after peer review.

- The final published version features the final layout of the paper including the volume, issue and page numbers.

Link to publication

\footnotetext{
General rights

- You may freely distribute the URL identifying the publication in the public portal. follow below link for the End User Agreement:

www.tue.nl/taverne

Take down policy

If you believe that this document breaches copyright please contact us at:

openaccess@tue.nl

providing details and we will investigate your claim.
}

Copyright and moral rights for the publications made accessible in the public portal are retained by the authors and/or other copyright owners and it is a condition of accessing publications that users recognise and abide by the legal requirements associated with these rights.

- Users may download and print one copy of any publication from the public portal for the purpose of private study or research.

- You may not further distribute the material or use it for any profit-making activity or commercial gain

If the publication is distributed under the terms of Article 25fa of the Dutch Copyright Act, indicated by the "Taverne" license above, please 


\title{
Rotation of a strongly magnetized hydrogen plasma column determined from an asymmetric Balmer- $\beta$ spectral line with two radiating distributions
}

\author{
A. E. Shumack, ${ }^{1}$ V. P. Veremiyenko, ${ }^{1}$ D. C. Schram, ${ }^{1,2}$ H. J. de Blank, ${ }^{1}$ W. J. Goedheer, ${ }^{1}$ H. J. van der Meiden, ${ }^{1}$ \\ W. A. J. Vijvers, ${ }^{1}$ J. Westerhout, ${ }^{1}$ N. J. Lopes Cardozo, ${ }^{1,2}$ and G. J. van Rooij ${ }^{1}$ \\ ${ }^{1}$ FOM-Institute for Plasma Physics Rijnhuizen, Association EURATOM-FOM, Trilateral Euregio Cluster, Nieuwegein, The Netherlands \\ ${ }^{2}$ Eindhoven University of Technology, Eindhoven, The Netherlands
}

(Received 1 June 2008; published 27 October 2008)

\begin{abstract}
A potential buildup in front of a magnetized cascaded arc hydrogen plasma source is explored via $\vec{E} \times \vec{B}$ rotation and plate potential measurements. Plasma rotation approaches thermal speeds with maximum velocities of $10 \mathrm{~km} / \mathrm{s}$. The diagnostic for plasma rotation is optical emission spectroscopy on the Balmer- $\beta$ line. Asymmetric spectra are observed. A detailed consideration is given on the interpretation of such spectra with a two distribution model. This consideration includes radial dependence of emission determined by Abel inversion of the lateral intensity profile. Spectrum analysis is performed considering Doppler shift, Doppler broadening, Stark broadening, and Stark splitting.
\end{abstract}

DOI: 10.1103/PhysRevE.78.046405

PACS number(s): 52.70. $-\mathrm{m}, 52.50 .-\mathrm{b}, 52.75 .-\mathrm{d}$

\section{INTRODUCTION}

A potential buildup at the exit of the magnetized cascaded arc hydrogen plasma source of the linear plasma generator Pilot-PSI has been shown to lead to high fluxes and a high source efficiency [1]. This has facilitated the production of unique plasma conditions. Pilot-PSI is a plasma generator for plasma surface interaction (PSI) studies in support of the international fusion experiment ITER $[2,3]$. It reproduces the hydrogen plasma conditions expected in the ITER divertor. Particle fluxes of up to $10^{24} \mathrm{~m}^{-2} \mathrm{~s}^{-1}$ are obtained with energy fluxes up to $10 \mathrm{MW} / \mathrm{m}^{2}$ and electron temperatures of $1-5 \mathrm{eV}$.

The cause of the potential buildup at the exit of the source is the source current path, which in contrast to similar linear plasma devices does not lie entirely within the source itself. The path of the electron current from the cathodes must cross the magnetic field in the radial direction to reach the anode. Due to magnetic confinement, the plasma resistivity in the radial direction is higher than in the axial direction. To follow the path of least resistance, the electron current spreads itself out in the axial direction until the effective radial resistance is the same as the axial resistance. Electric fields associated with the current path drive plasma rotation via an $\vec{E}$ $\times \vec{B}$ force.

$\vec{E} \times \vec{B}$ drift rotation has been well studied in the literature [4-8]. As already indicated in [1], rotation velocities in PilotPSI can be up to $10 \mathrm{~km} / \mathrm{s}$, one order of magnitude higher than expected from the ambipolar fields. This paper reports on the measurements of plasma rotation, which form the basis of an exploration of the potential buildup in front of the source.

The plasma rotation is studied by optical emission spectroscopy (OES). Direct passive spectroscopy is not possible for hydrogen ions which do not radiate. Instead we analyze the light emitted by the neutral atoms. This approach was successfully followed by others (e.g., Meyer et al. [6]) by virtue of an efficient coupling of the excited atoms to the ions via fast symmetric charge exchange.
Asymmetry is observed in the Balmer- $\beta$ spectra measured for the high density, magnetized hydrogen plasma under study. Asymmetric spectra have been observed earlier $[4,7,9-11]$ and analyzed with the assumption of two radiating populations. The assumption of two distributions is given a thorough consideration in this paper for the plasma conditions present.

The method of spectrum analysis is assessed on the basis of the residue of the fitting procedure and the plasma densities and temperatures that it predicts. Thomson scattering measurements form the basis of quantitative comparison.

In Sec. II, the plasma device is described and the experimental conditions given. Section III presents the optical emission spectroscopy measurements, explains the method of spectrum analysis and gives the results. Section IV explores the mechanisms behind the light production and the shape of the spectral emission line. The rotation of the plasma in relation to the radial electric field which causes it is discussed in Sec. V.

\section{EXPERIMENT}

\section{A. Linear plasma generator Pilot-PSI}

The hydrogen plasma under study in this work was produced in the linear plasma generator Pilot-PSI [1]. Figure 1 shows the experimental arrangement of Pilot-PSI and the emission spectroscopy diagnostic.

Plasma is produced with a cascaded arc, see Fig. 2. Its design is based on extensive work at the Eindhoven University of Technology $[12,13]$.

Hydrogen flows at 2.5 standard liters per minute or 1.0 $\times 10^{21} \mathrm{H}_{2} / \mathrm{s}$ through a discharge channel, $32 \mathrm{~mm}$ long and $4 \mathrm{~mm}$ in diameter. A discharge current is stabilized to $80 \mathrm{~A}$ between three tungsten cathodes and a 5-8 $\mathrm{mm}$ diameter copper-tungsten nozzle that serves as the anode. Unless stated otherwise, the nozzle diameter for the measurements presented in this paper is $8 \mathrm{~mm}$. The plasma expands out of the high pressure source $\left(10^{4} \mathrm{~Pa}\right.$ in the cathode region) into a $1 \mathrm{~m}$ long, $40 \mathrm{~cm}$ diameter vacuum vessel. During operation, 


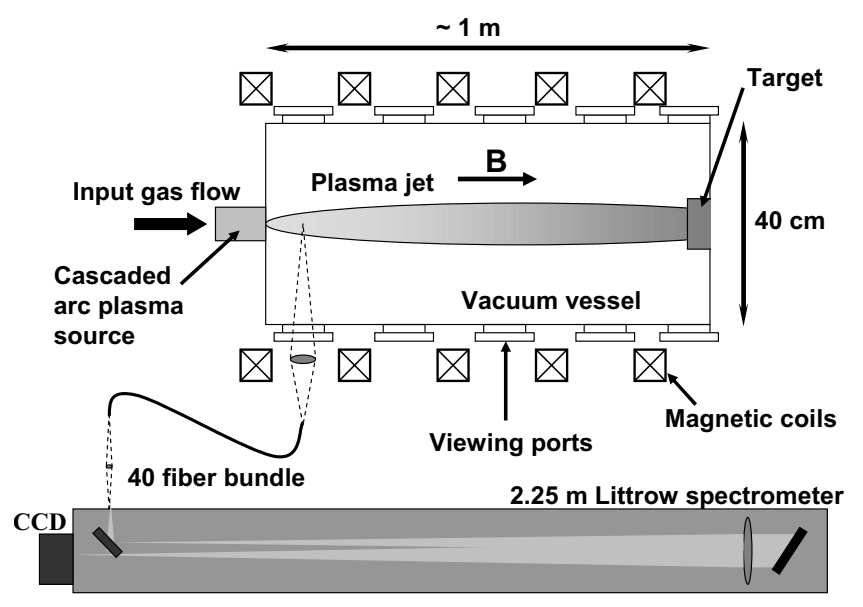

FIG. 1. Schematical drawing of the experimental arrangement of Pilot-PSI and its optical emission spectroscopy diagnostic.

the background pressure is typically $1-5 \mathrm{~Pa}$. An axial magnetic field directed away from the source with a variable strength in steps of $0.4 \mathrm{~T}$ to a maximum of $1.6 \mathrm{~T}$ confines the plasma to form a jet. The magnetic field duration is limited by the cooling of the coils and is set to 30 seconds for $0.4 \mathrm{~T}$ and 3 seconds for higher fields. The plasma beam extends to the opposite end of the vessel where it impinges on a water-cooled target. The potential distribution over the cascaded arc plates is shown in Fig. 3 for different magnetic field values. Without magnetic field, the $130 \mathrm{~V}$ discharge potential is distributed evenly over all plates. A magnetic field only affects the potential difference between the anode and the first cascaded plate. The electron temperature $\left(T_{e}\right)$ and density $\left(n_{e}\right)$ of the plasma are measured with Thomson scattering at $40 \mathrm{~mm}$ from the nozzle of the source. Full details of this diagnostic can be found in [14]. Thomson scattering is performed with a $532 \mathrm{~nm} \mathrm{Nd:YAG} \mathrm{second} \mathrm{harmonic} \mathrm{laser} \mathrm{at}$ $10 \mathrm{~Hz}$ with $0.4 \mathrm{~J} /$ pulse. The signal is averaged over the duration of the magnetic field pulse. A fiber array is used to collect the scattered light so that the entire plasma diameter is observed simultaneously in 40 channels. The Thomson scattering spectra are absolutely calibrated with Rayleigh

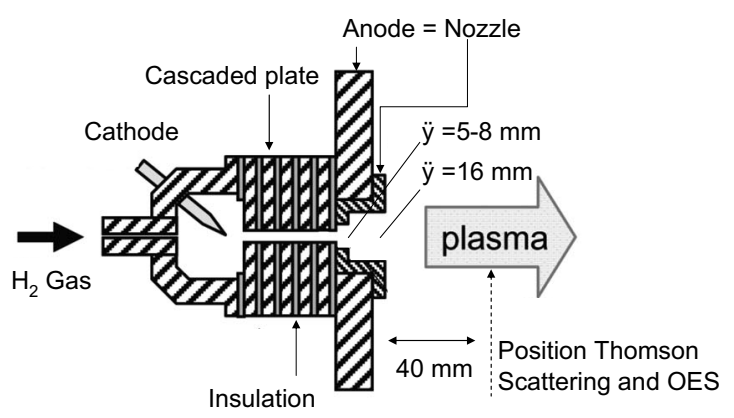

FIG. 2. The cascaded arc plasma source. The working gas enters the plasma source via the gas inlet. It is ionized in the arc channel of the cascaded plates due to the discharge between the three cathodes and the anode. The nozzle is electrically connected to anode which is attached to the vessel wall. Plasma exits the source through the nozzle. Thomson scattering and OES measurements are performed at $40 \mathrm{~mm}$ from the nozzle.

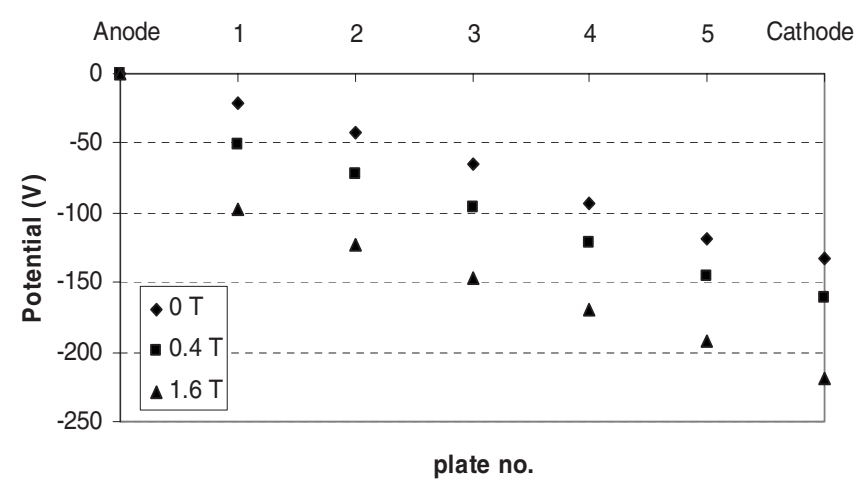

FIG. 3. A potential distribution over cascaded plates in a hydrogen plasma. In a magnetic field the voltage over the arc is significantly higher. The extra voltage drops mainly between the anode and the first plate.

scattering on argon in the vessel at a pressure of $50 \mathrm{~Pa}$. The electron density and temperature profiles at the four magnetic field settings discussed in this paper are shown in Fig. 4. The electron density increases with increasing magnetic field because of improved plasma confinement. This can also be seen in the narrowing of the density profiles. The influence of the magnetic field on the temperature profile is
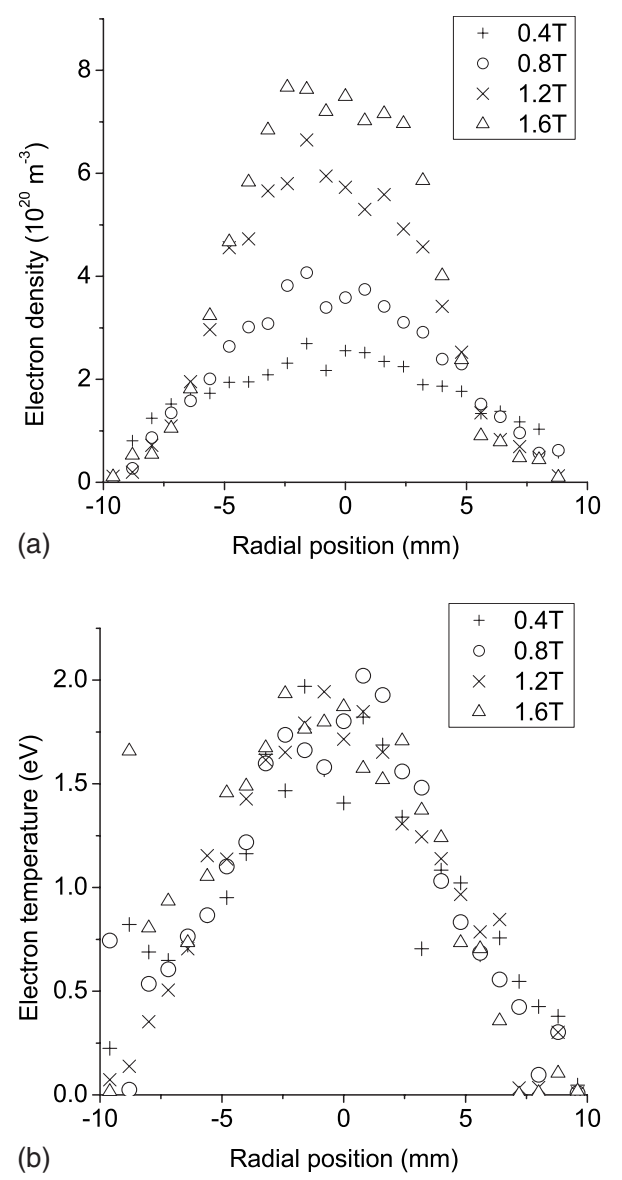

FIG. 4. The radial electron (a) density and (b) temperature profiles of the hydrogen plasma jet measured with Thomson scattering at $40 \mathrm{~mm}$ from the source in a magnetic field of $0.4,0.8,1.2$, and $1.6 \mathrm{~T}$. 


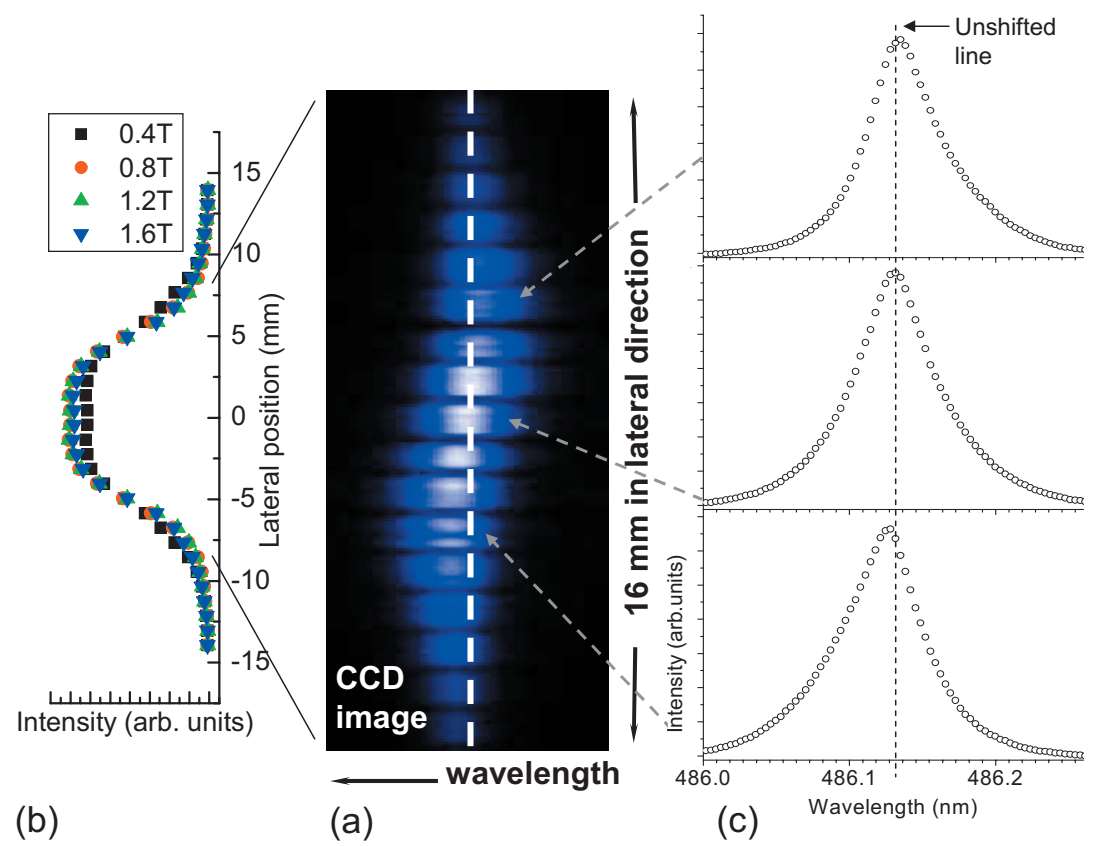

FIG. 5. (Color) A typical OES measurement at a magnetic field strength of 1.6 T. (a) Part of a raw CCD-image of the spectrally as well as spatially resolved Balmer- $\beta$ light, indicating plasma rotation. The 17 bands of light correspond to individual optical fibers. The spectral line appears shifted to the red at the bottom of the plasma jet and to the blue at the top. At the jet edges it is unshifted. The spectral shift reveals rotation of the radiating species. The maximum Doppler shift from the central wavelength value is about 5 pixels. This corresponds to a velocity of $9.3 \mathrm{~km} / \mathrm{s} \pm 10 \%$ (b) The corrected lateral intensity profile displaying a flat top, pointing to a hollow emission profile. (c) Spectral profiles from, respectively, above, on, and under the jet axis. The off-center profiles are clearly asymmetric, with the direction of asymmetry depending on the lateral position. In the jet center, the profile is symmetric.

minimal. For a discussion of these plasma conditions, see Ref. [1].

\section{B. Optical emission spectroscopy setup}

The OES arrangement is depicted in Fig. 1. Plasma light is collected at an axial distance of $40 \mathrm{~mm}$ from the nozzle of the source with a lens (diameter $6 \mathrm{~cm}$, focal length $20 \mathrm{~cm}$ ) in the direction perpendicular to the jet axis, at magnification $M=0.4 \times$. A polarizer selects $\pi$ components of Zeemansplitted spectral lines. An array of 40 individual $0.4 \mathrm{~mm}$ diameter quartz fibers relays the collected light to an in-house constructed, single-pass spectrometer in Littrow configuration. In this way, spatial information is preserved and a spectral range of approximately $1 \mathrm{~nm}$ can be investigated over the entire $2-3 \mathrm{~cm}$ width of the plasma jet profile simultaneously.

The output of the fiber array is imaged on an adjustable slit. Behind the slit, light is collimated by an achromatic lens of $15 \mathrm{~cm}$ in diameter with a focal length of $2.25 \mathrm{~m}$. The blazed diffraction grating is optimized for the second diffraction order (blaze angle $17.45^{\circ}$ ). It has dimensions of 11 $\times 11 \mathrm{~cm}$ and a groove density of 1200 per $\mathrm{mm}$. The signal is detected on a $298 \times 1152$ pixel CCD (charged coupled device) camera. The instrument function of the spectrometer, determined with He-Ne laser light, has a width smaller than $0.003 \mathrm{~nm}$ (full width at $1 / e$ ), corresponding approximately to 1 pixel at the wavelength of the Balmer- $\beta$ transition.

The exposure time of the CCD camera is several seconds, the maximum time allowed by the duration of the magnetic field pulse. Fast temporal fluctuations are thus averaged. These fluctuations were studied in an independent experiment using a photodiode. Variations of typically $\pm 30 \%$ with respect to the mean value were observed with a frequency of close to $1 \mathrm{MHz}$.

This paper reports on spectroscopy data taken at one axial position, at $4 \mathrm{~cm}$ from the nozzle of the plasma source. Although not presented in this paper, the plasma parameters do display axial dependence. The ion rotation velocity decreases in the axial direction with a characteristic decay length of about one-half meter. The reason for the decrease is the penetration depth of the electric field which causes it, as explained in Sec. V. The temperature and density also decrease slowly along the axis due to plasma recombination.

\section{SPECTRUM ANALYSIS}

In this section we develop the methodology for interpretation of the OES data. We start with a detailed consideration of a representative measurement.

\section{A. Measured Balmer- $\beta$ line shape and intensity profile}

Figure 5(a) shows a typical OES measurement at a magnetic field of 1.6 T. Here, we see part of a raw CCD image of the spatially (vertical axis) as well as spectrally (horizontal axis) resolved Balmer- $\beta$ line, indicating plasma rotation. The complete width of the CCD image corresponds approximately to $1 \mathrm{~nm}$ around the wavelength of the Balmer- $\beta$ line. Only the central part of $\sim 0.35 \mathrm{~nm}$ is shown in the figure. 

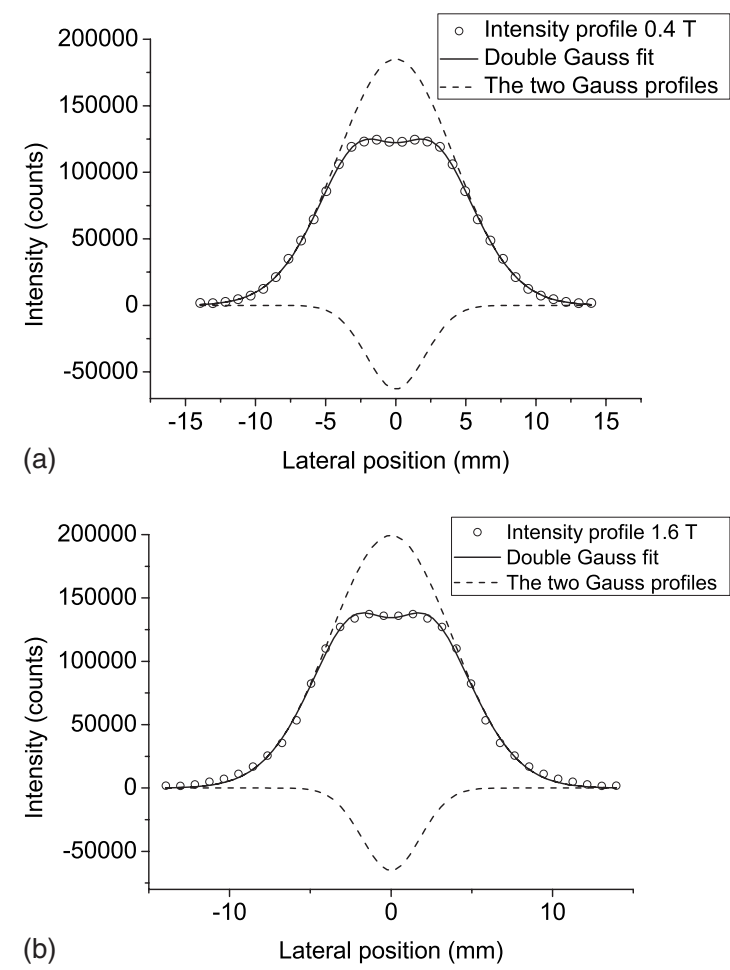

FIG. 6. Lateral intensity profiles at (a) $0.4 \mathrm{~T}$ and (b) $1.6 \mathrm{~T}$. Data is fit with the sum of two Gauss profiles, one of which is inverted.

The vertical axis is the spatial coordinate, in the lateral direction (perpendicular the plasma propagation), spanning approximately $40 \mathrm{~mm}$, about $16 \mathrm{~mm}$ of which is shown in the figure. We call this the lateral direction, distinguishing it explicitly from the radial direction because the measurement is a line of sight integration. The different bands in the CCD image correspond to the individual fibers in the array. The finer structures within these bands are due to imperfections in the spectrometer slit. The spectral lines appear Doppler shifted to the red at the bottom of the plasma jet and to the blue at the top, indicating rotation of the radiating species. Towards the edges of the beam the rotation is damped and the profile is unshifted. The direction of rotation is clockwise as viewed from the source, looking towards the target.

We can estimate the value of the maximum rotation velocity at $1.6 \mathrm{~T}$ from Fig. 5(a). We focus on the off-center fiber images with the largest wavelength shift. Their entire Doppler broadened band appears to be shifted from the central line by approximately 5 pixels. This corresponds to a Doppler shift of $0,015 \mathrm{~nm}$ at the wavelength of the Balmer- $\beta$ transition and a velocity of $9.3 \mathrm{~km} / \mathrm{s} \pm 10 \%$.

Figure 5(b) shows the lateral intensity profile corresponding to the entire CCD image. This was obtained by integration over the wavelength axis of the CCD image and over the spatial band corresponding to each fiber. The intensity profiles measured at other magnetic field strengths are plotted on the same graph. We corrected for variations in the transmittance of the individual fibers. A cutoff in the spectrometer throughput at the lower fibers (left-hand side of the profile) was corrected for by mirroring the right-hand side of the profile to the left.

We observe that the intensity profiles do not change significantly as the magnetic field and thereby also the electron

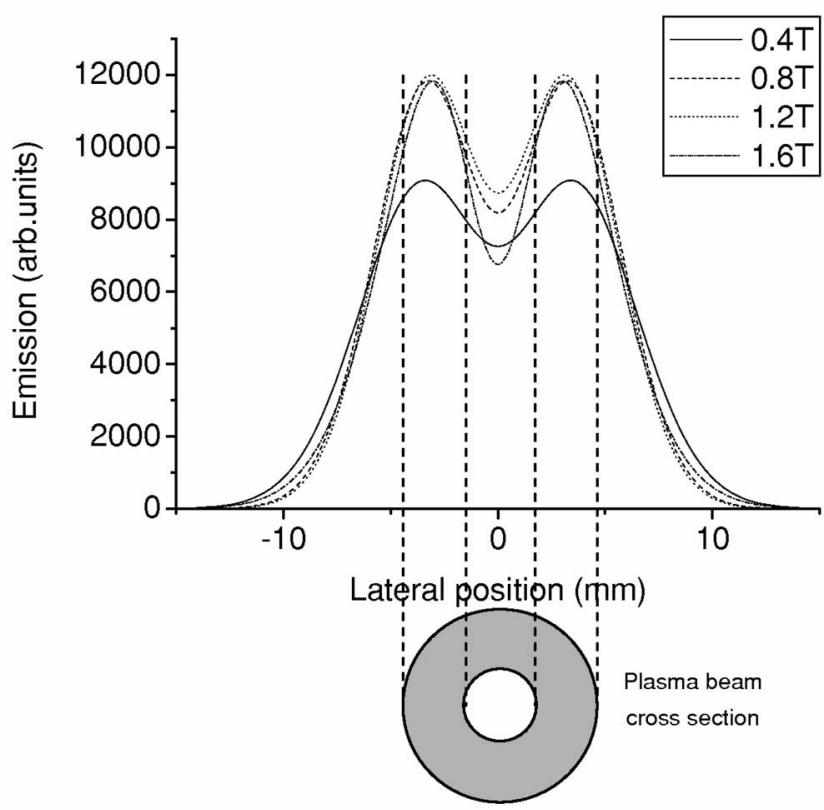

FIG. 7. Radial emission profiles for the four magnetic field values, obtained by Abel inverting the fit of the intensity profiles. Emission profiles are clearly hollow in the center of the plasma beam. A schematic cross section of plasma beam is shown indicating positions of strongest emission by shading.

density is varied. Furthermore, the profiles are flat in the center which points to a hollow emission profile. Abel inversions of the intensity profile are given in Sec. III B, along with the implications of a hollow emission profile shape. The mechanisms leading to the hollow emission profiles are set out in Sec. IV B.

In Fig. 5(c) typical spectral emission lines are shown corresponding to different lateral positions. The line profile in the middle of the plasma column is symmetric, off-center the profiles are shifted and become asymmetric. This asymmetry is caused by the presence of two populations of radiating (excited) neutrals, one hot, rotating and coupled to the ions, the other cold and only slowly rotating. The profiles can hence be fit with a double Voigt function to obtain information on rotation velocity, temperature, and density. The fit methodology is described in Sec. III C.

\section{B. Hollow emission profile}

Figures 6(a) and 6(b) show the lateral intensity profiles for a magnetic field of $0.4 \mathrm{~T}$ and $1.6 \mathrm{~T}$, respectively. We perform a fit of the profiles with the sum of two Gaussian profiles, one of which is inverted. The fit approximates the edges of the intensity profile better for $0.4 \mathrm{~T}$ than for $1.6 \mathrm{~T}$. Abel inversion of the lateral intensity profile gives the radial emission profile. Since Gaussian functions are invariant under Abel inversion [15], the chosen fit function allows for simple analysis. Abel inversion does not affect the shape of the Gaussian functions themselves, only changes the ratio between them. We see the result in Fig. 7 for all four magnetic field values. The profiles are clearly hollow. In Sec. 

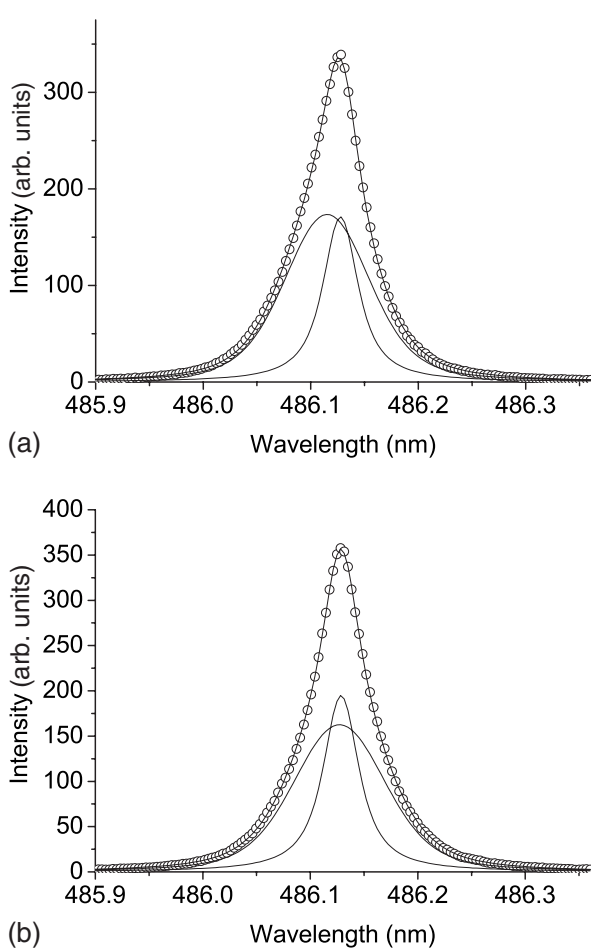

FIG. 8. A double Voigt fit of spectra taken at $1.6 \mathrm{~T}$ from (a) below the jet axis (b) on the jet axis. The two single Voigt components are shown, one corresponding to the hot, rotating population, the other to the cold and almost nonrotating population.

IV B we show that this can be expected from a balance of the plasma processes present.

The practical consequence of the hollow emission profile is that the strongest light emission does not occur at the center of the jet where the electron density is highest. As shown in Fig. 7, most light collected is emitted from a ring, at a position where the electron density and temperature are substantially lower than the maximum value.

\section{Asymmetric line profile: Double Voigt fit}

In Sec. III A, Fig. 5(c) we see that the emission profiles above and below the plasma axis are asymmetric. The direction of this asymmetry is reversed for profiles recorded above the plasma jet axis as compared to those recorded below the axis. We fitted these profiles assuming two populations of radiating hydrogen atoms, a procedure also followed by $[7,10,11]$, see Fig. 8 . One population is coupled to the ions. It is hot, with a temperature of several $\mathrm{eV}$, and rotates at high speed, up to $10000 \mathrm{~m} / \mathrm{s}$. The other is cold -0.1 to $0.5 \mathrm{eV}$ - and rotates only very slowly, at maximum $10 \%$ of the velocity of the hot component.

We fitted the spectrum from each population with a Voigt profile, the convolution between a Gauss and a Lorentz profile. This is required for the plasma densities and temperatures in our plasma, where both Doppler broadening (Gaussian) and Stark broadening (Lorentzian) of the Balmer- $\beta$ spectral line are important. For example, at a temperature of $1 \mathrm{eV}$ and a density of $10^{20} \mathrm{~m}^{-3}$ the Gaussian from Doppler broadening has a FWHM (full width half maximum) of
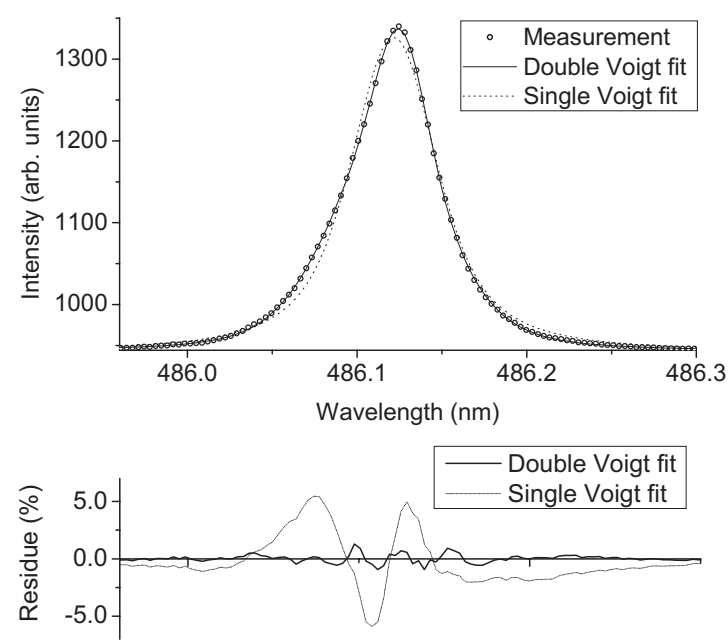

FIG. 9. An off-center asymmetric Balmer- $\beta$ line fitted with a single and a double Voigt profile $(B=1.2 \mathrm{~T})$. The residues for the double Voigt fit are significantly smaller and more stochastic.

$37.4 \mathrm{pm}$. The Lorentz profile from Stark broadening of the Balmer- $\beta$ line has a FWHM of 36.6 pm [16].

In our double Voigt fitting procedure, eight parameters are left free: These are the baseline (offset), the amplitudes (or areas) of the two Voigt components, their Doppler widths and one Lorentz width (the same for both), the exact position of the cold component and the shift of the hot component with respect to the cold one. We take the same Lorentz width for both components because total free fits return equal values for these two fit parameters. Also, the ratio between the amplitudes of the hot and cold components remains constant along the lateral direction indicating that the emission from both components originates from the same position. From the double Voigt fit we are able to obtain the ion rotation velocity and temperature from, respectively, the spectral shift and Doppler width of the hot component. The electron density is determined from the Lorentz width, but as we will see in Sec. III E this quantity is not well determined.

A double Voigt fit shows improvement compared to a single Voigt fit as seen in Fig. 9. The residues are smaller, around $1 \%$, and more stochastic. The remaining structure in the very small residue can be understood as an artifact of the instrument function.

\section{Results and discussion for rotation and ion temperature}

Figure 10 shows the results for the ion rotation. Rotation velocities of up to $10000 \mathrm{~m} / \mathrm{s}$, are obtained for the highest magnetic field strength, 1.6 T. This is conform to estimates from the raw CCD image in Fig. 5(a). As shown in Sec. V electric field strengths are available to drive $\vec{E} \times \vec{B}$ rotation of this magnitude.

The peak rotation velocities have values up to one-half of the ion acoustic velocity in which range ion viscous heating $[5,7,17]$ becomes significant. Ion neutral friction and charge exchange are responsible for damping of the rotation towards the edges of the beam while heating the neutrals. This enhancement of velocity shear significantly enhances ion viscous heating. 


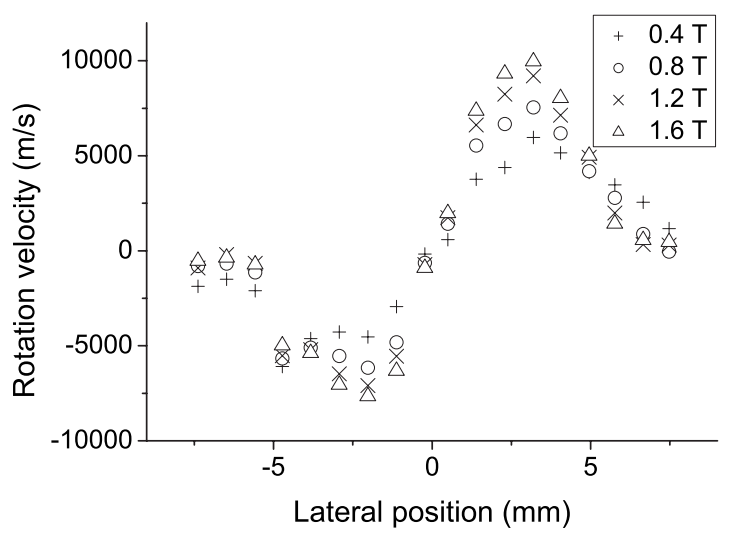

FIG. 10. Lateral profiles of rotation velocity determined from a double Voigt fit of the Balmer- $\beta$ line shape at magnetic field strengths of $0.4 \mathrm{~T}, 0.8 \mathrm{~T}, 1.2 \mathrm{~T}$, and $1.6 \mathrm{~T}$.

Ion temperatures are indeed somewhat higher than the electron temperature given in Fig. 4. The results of the double Voigt fit for ion temperature are given in Fig. 11.

We estimate the strength of the viscous heating to determine whether it is indeed an important heating channel. The viscous heating term is given by Braginskii [18],

$$
Q_{\mathrm{visc}}=-\Pi_{\alpha \beta} \frac{\partial v_{\alpha}}{\partial x_{\beta}},
$$

where $\Pi_{\alpha \beta}$ is the stress tensor. We take $\partial v_{\theta} / \partial R$ to be the largest systematic component. For Hall factor $\omega_{c i} \tau_{i i} \sim 1$, true for all plasma conditions under study, this term is of the order

$$
Q_{\mathrm{visc}} \sim n_{i} k_{B} T_{i} \tau_{i i}\left(\frac{v_{i \theta}(R)}{R}\right)^{2}=\frac{e \hat{T}_{i}^{5 / 2} 2 \times 10^{13} \Omega_{i}^{2}}{\ln \Lambda},
$$

where we have approximated $\partial v_{i \theta}(R) / \partial R$ by $v_{i \theta}(R) / R . n_{i}$ is the ion density $\left(\mathrm{m}^{-3}\right), k_{B}$ is Boltzmann's constant $(\mathrm{J} / \mathrm{K}), T_{i}$ is the ion temperature $(\mathrm{K}), \tau_{i i}$ is the ion-ion collision time $(\mathrm{s})$, $v_{\theta}$ is the azimuthal velocity $(\mathrm{m} / \mathrm{s}), R$ is the radius $(\mathrm{m}), e$ is

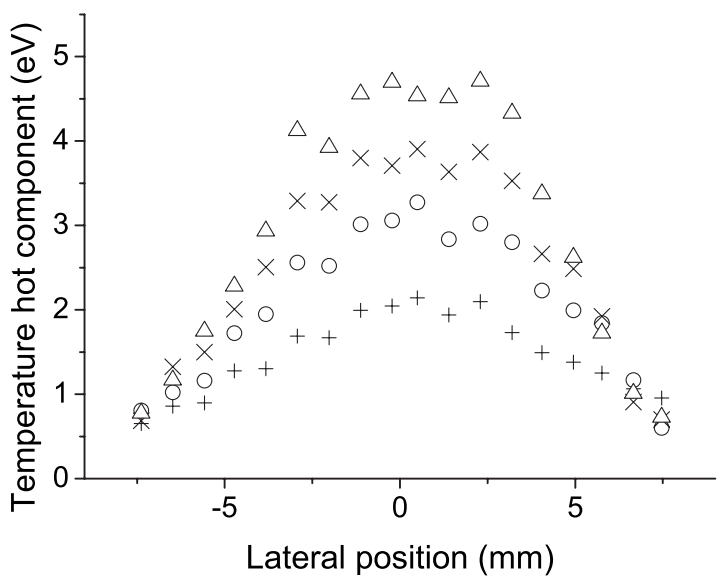

FIG. 11. Lateral profiles of ion temperature determined from a double Voigt fit of the Balmer- $\beta$ line shape at magnetic field strengths of $0.4 \mathrm{~T}, 0.8 \mathrm{~T}, 1.2 \mathrm{~T}$, and $1.6 \mathrm{~T}$.

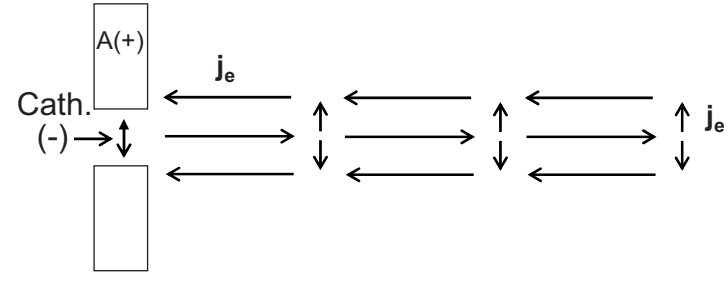

FIG. 12. The electrons cannot close the circuit within the source due to their limited mobility across the magnetic field. The radial electron current path spreads out in the axial direction until the resistance of the axial and radial current paths are equal.

electron charge $(\mathrm{C}), \hat{T}_{i}$ is the ion temperature $(\mathrm{eV}), \Omega$ is the rotation frequency $(\mathrm{rad} / \mathrm{s})$, and $\ln \Lambda$ is the Coulomb logarithm. Filling in values for the off axis plasma, $r=2 \mathrm{~mm}$, at a magnetic field of $1.2 \mathrm{~T}$ gives $Q_{\text {visc }}$ in the order of $10^{8} \mathrm{~W} / \mathrm{m}^{3}$.

We can judge the importance of viscous heating at the edge of the plasma by comparison with the central heat input by ohmic loss,

$$
Q_{\text {ohmic }}=\sigma_{\|} E_{z}^{2},
$$

where $\sigma_{\|}$is the conductivity parallel to the magnetic field and $E_{z}$ is the axial electric field. Because of magnetic field confinement, the source plasma current penetrates into the vessel as shown in Fig. 12. The penetration depth is about one-half meter. This current is associated with a strong radial electric field $E_{r}$ and a weak axial electric field $E_{z}$. These fields are discussed in more detail in Sec. V. For an estimation of $E_{z}$, we assume an axial potential drop of $50 \mathrm{~V}$ (close to one-half of the source potential). This falls over one-half meter, giving $E_{z}=100 \mathrm{~V} / \mathrm{m}$. The conductivity from Spitzer [19] is

$$
\sigma_{\|}=\frac{2 \times 10^{4} T_{e}^{\hat{3} / 2}}{\ln \Lambda} .
$$

The result is an Ohmic heating of about $10^{8} \mathrm{~W} / \mathrm{m}^{3}$, of the same order as viscous heating at the edges.

Hence, we conclude that ion viscous heating can be important, the occurrence of which agrees with the observed high ion temperatures.

\section{E. Assessment of fit based on density and temperature predictions}

From Abel inversion of the intensity profiles shown in Fig. 7 we know that the radial emission profile of the plasma is hollow. We therefore expect the densities resulting from the fit of the spectra to be lower than the peak electron densities measured by Thomson scattering. This is because most emission originates from a ring towards the edges of the beam where the electron density is lower.

There is uncertainty in the position of the emission ring as determined by Abel inversion. The analysis is very sensitive to the asymptotic behavior of the intensity profile for $r \rightarrow \infty$. Also, the instrument function provides an extra broadening of the profile which is more significant for the narrower inverse Gauss profile than for the broader Gauss profile. Accounting for this effect could either shift the emission ring in 
(a)

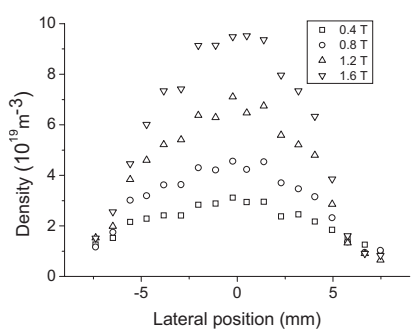

(b)

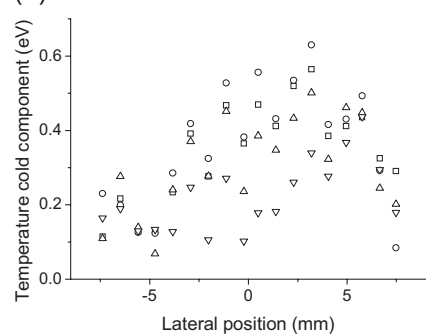

FIG. 13. Lateral profiles of (a) electron density and (b) temperature of the cold neutral population determined from a double Voigt fit of the Balmer- $\beta$ line shape at magnetic field strengths of $0.4 \mathrm{~T}$, $0.8 \mathrm{~T}, 1.2 \mathrm{~T}$, and $1.6 \mathrm{~T}$.

the Abel inverted profile to larger or smaller radii.

Keeping this uncertainty in mind, we consider the densities that could be expected from spectra originating from an emission ring at the position determined from Abel inversion. This radial position is approximately $2-6 \mathrm{~mm}$, see Fig. 7. Here, the electron density from Thomson scattering ranges from $2 \times 10^{20} \mathrm{~m}^{-3}$ up to almost the maximum electron density for each magnetic field strength.

Other densities can be expected from a consideration of the processes behind the formation of a hollow emission profile. Based on a reaction balance, we predict emission to originate from a plasma position with densities ranging between 0.5 and $1.5 \times 10^{20} \mathrm{~m}^{-3}$. This is explained in Sec. IV B.

Results for the electron density from the double Voigt fit are shown in Fig. 13(a). The peak electron densities lie in the range between $2 \times 10^{19} \mathrm{~m}^{-3}$ and $1 \times 10^{20} \mathrm{~m}^{-3}$. Especially for low magnetic fields, this is somewhat lower than expected. There are several mechanisms that could explain this.

It is well known that the profile for a purely Stark broadened Balmer- $\beta$ profile deviates from a single Lorentzian because of Stark splitting [20,21]. It exhibits structure at its peak (a dip), and thus seems broader than a single Lorentz. If one attempts to fit such a profile with a Voigt function, the Stark part may be partly interpreted as Gaussian because of this extra structure. This effect means that a Gaussian and Stark broadened profile fitted with a double Voigt function may overestimate the Doppler widths and underestimate the Stark width, thus underestimating the electron density. We confirmed that this is a possibility by fitting high density Balmer lines from the literature with a Voigt function. An example is the Balmer- $\beta$ line from Thomson and Helbig [16] at a density of $8 \times 10^{20} \mathrm{~m}^{-3}$ and a temperature of $13500 \mathrm{~K}$. We would expect a Voigt fit of this profile to return a mostly Lorentzian broadened profile. The result of such a fit is in fact a Gaussian component with a width of $137 \mathrm{pm}$ and a Lorentzian width of only $60 \mathrm{pm}$.

The misinterpretation of part of the Stark profile must of course also have consequences for the ion temperature determination. The total width of the cold Gaussian component can give us an upper limit on the Gaussian interpretation of the density. Here we also take into account the instrument function and a minimum background gas temperature. From the upper limit determined, we conclude that Gaussian interpretation of the density will not cause overestimation of the ion temperature by more than $10 \%$.
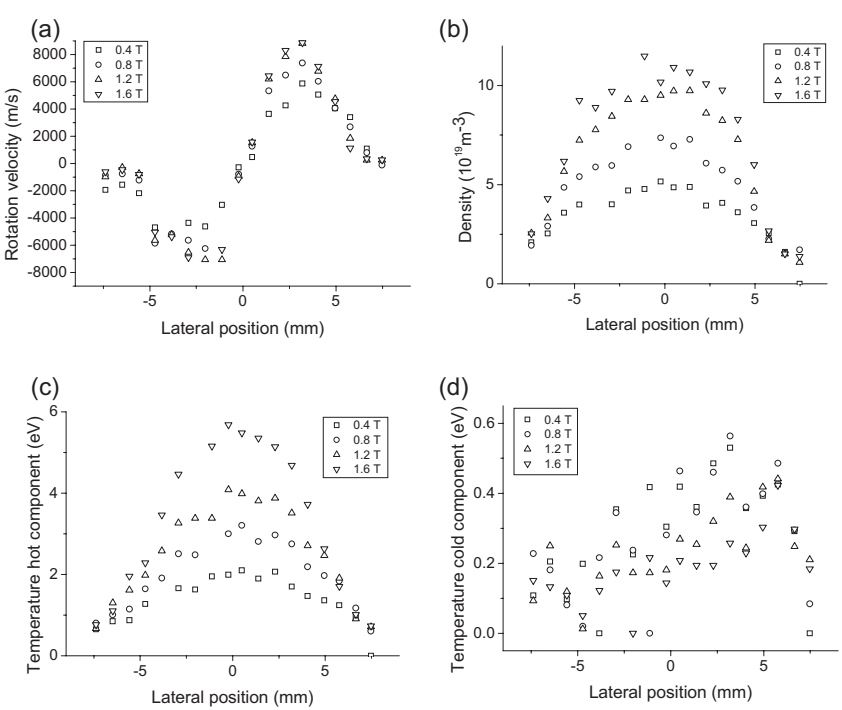

FIG. 14. Results from a double Voigt fit of the Balmer- $\beta$ line shape in which each Lorentz profile is replaced by a double Lorentz profile in order to simulate Stark splitting. The lateral profiles shown are of the (a) rotation velocity, (b) electron density, (c) ion temperature, and (d) cold neutral population.

To investigate the influence of Stark splitting on the fit, we simulate it by replacing each of the Lorentz components of the double Voigt fit function with two Lorentz profiles shifted with respect to one another. The distance $d$ separating a pair of Lorentz profiles is set at a multiple of the half-width at half-maximum (HWHM) of a single Lorentz component. This multiple, $d$, is determined from fits of profiles traced from the literature $[16,20]$ and from fits of spectral profiles from the Pilot-PSI plasma. The best fits result from a shift $d$ of 1.0 times the HWHM. Such a fit of the Balmer- $\beta$ spectrum measured by Helbig and Nick [20] for a density of $10.8 \times 10^{22} \mathrm{~m}^{-3}$ gives a HWHM of each Lorentz profile of $1.370 \mathrm{~nm}$. This conversion factor between density and Lorentz width, in combination with experimental scaling $w_{L}(\mathrm{HWHM}) \propto n^{2 / 3}$ from [22], is the basis of density determination in fits with Stark splitting simulation.

As seen in Fig. 14 the density determined from the fit with Stark splitting was higher than without splitting. The values corresponding to the highest and lowest field also lie closer together. Peak densities of $4 \times 10^{19} \mathrm{~m}^{-3}$ to $1.1 \times 10^{20} \mathrm{~m}^{-3} \mathrm{re}-$ sulted. The residue of the fit remained below $1 \%$. The temperature and rotation were also affected, with a greater spread in the temperature parameter over the different magnetic field values and a smaller spread in the rotation. We note here that the value of the rotation velocity with and without Stark splitting remains the same within about $10 \%$, emphasizing the suitability of the spectral analysis for rotation velocity determination.

The temperature of the cold component, shown in Fig. 13 is typically $\sim 0.2-0.4 \mathrm{eV}$. This is reasonable for a background gas in close proximity to a hot plasma. This temperature may be somewhat overestimated because of a partial Gauss interpretation of the density. 


\section{CONSIDERATION OF UNDERLYING PLASMA PROCESSES}

\section{A. $H^{*}(n=4)$ production mechanisms}

Molecular activated recombination (MAR) [23-25] is a significant production path of excited neutrals under the Pilot-PSI plasma conditions. First, a molecular ion is produced via charge exchange between an ion and a rovibrationally excited background gas molecule,

$$
\mathrm{H}^{+}+\mathrm{H}_{2}(r, \nu) \rightarrow \mathrm{H}(1 s)+\mathrm{H}_{2}^{+}(r, \nu) .
$$

This is followed by the fast dissociative recombination of the molecular ion resulting in two hydrogen atoms one of which is carrying an excess of internal energy and ends up in at least the $n=2$ level, or most probably in $n=3$ [26]:

$$
\mathrm{H}_{2}^{+}(r, v)+e^{-} \rightarrow \mathrm{H}^{*}+\mathrm{H}(1 s) .
$$

$\mathrm{H}(n=4)$ is produced by direct fast electron excitation from the $n=2$ and $n=3$ levels,

$$
\mathrm{H}^{*}(n=2,3)+e^{-} \rightarrow \mathrm{H}^{*}(n=4)+e^{-} .
$$

For the prevailing conditions with $n_{e}=n_{i}$ of the same order as $n_{\mathrm{H}_{2}}$, the first step (5) in the MAR sequence is the slowest one and sets the rate of MAR. At an electron temperature of $1-2 \mathrm{eV}$, the rate is $4-7 \times 10^{-17} \mathrm{~m}^{3} \mathrm{~s}^{-1}$ [26]. This rate can be 100 times higher if the hydrogen molecule is vibrationally excited with $v \geqslant 4[27]$; around $4-7 \times 10^{-15} \mathrm{~m}^{3} \mathrm{~s}^{-1}$.

Other possible $\mathrm{H}^{*}$ production mechanisms have lower rates. The rate of direct electron excitation from the ground state to $n=2$ is $10^{-17} \mathrm{~m}^{3} \mathrm{~s}^{-1}$ at $1 \mathrm{eV}$ and $10^{-16} \mathrm{~m}^{3} \mathrm{~s}^{-1}$ at $2 \mathrm{eV}$. This is in contrast to comparable linear plasma devices, Pisces $\left(T_{e}=3-35 \mathrm{eV}\right)$ [28], NAGDISI $\left(T_{e}\right.$ $=5-10 \mathrm{eV})$ [29], and PSI-I $\left(T_{e}=5 \mathrm{eV}\right)$ [6], with much higher electron temperatures, where direct excitation is thus important. Three particle recombination having a small reaction rate, but with high densities available, will give a small contribution to the $\mathrm{H}^{*}$ production.

\section{B. Hollow emission profiles from reaction balance}

In this section we show how a hollow radial emission profile follows from the production mechanism of $n=4$ excited atoms and a balance of the reactions involved.

From Eqs. (5) and (6), radiation and electron deexcitation, the balance of population and depopulation processes for $\mathrm{H}_{2}{ }^{+}$ ions and $\mathrm{H}^{*}(n=3)$ excited atoms, respectively, can be written as

$$
\begin{gathered}
n_{\mathrm{H}^{+}} n_{\mathrm{H}_{2}} k_{c x}=n_{\mathrm{H}_{2}}+n_{e} k_{d r}, \\
n_{\mathrm{H}_{2}}+n_{e} k_{d r}=n_{\mathrm{H}(n=3)}\left(n_{e} K_{3}+A_{3}\right),
\end{gathered}
$$

with $k_{c x}$ and $k_{d r}$ denoting the rates of charge exchange and dissociative recombination, respectively, and $K_{3} \equiv \sum_{i \neq 3} k_{3 i}$, where $k_{i j}$ is the rate of (de)exitation from the $i$ th to the $j$ th level, $A_{3}=\sum_{i<3} A_{3 i}$ and $n \equiv$ density.

From reaction (7), radiation and electron deexcitation, the balance of $\mathrm{H}^{*}(n=4)$ is

$$
n_{\mathrm{H}(n=3)} n_{e} k_{34}=n_{\mathrm{H}(n=4)}\left(n_{e} K_{4}+A_{4}\right)
$$

with $K_{4} \equiv \sum_{i \neq 4} k_{4 i}$ and $A_{4} \equiv \Sigma_{i<4} A_{4 i}$.

If $n_{e}>A_{3} / K_{3}$ and $n_{e}>A_{4} / K_{4}$-true for $n_{e}>5 \times 10^{19} \mathrm{~m}^{-3}$ (data from $[30,31]$ ) — then we can eliminate $A_{3}$ and $A_{4}$. Assuming also that $K_{3} \simeq k_{34}$, we obtain a balance between underlying processes for $\mathrm{H}^{*}(n=4)$,

$$
n_{\mathrm{H}^{+}} n_{\mathrm{H}_{2}} k_{c x} \simeq n_{\mathrm{H}(n=4)} n_{e} K_{4} .
$$

Quasineutrality gives $n_{e}=n_{\mathrm{H}^{+}}$and thus

$$
n_{\mathrm{H}(n=4)} k_{c x} \simeq \frac{n_{\mathrm{H}_{2}} k_{c x}}{K_{4}} .
$$

At sufficiently high electron densities $\left(n_{e}>5 \times 10^{19} \mathrm{~m}^{-3}\right)$ deexcitation of the $\mathrm{H}^{*}(n=4)$ level outweighs radiation. The line emission thereby becomes independent of $n_{e}$ and depends only on $n_{\mathrm{H}_{2}}$. According to the Thomson scattering measurements shown in Fig. 4, this occurs at a radius of about $8 \mathrm{~mm}$.

The shape of the emission profile above an electron density of $5 \times 10^{19} \mathrm{~m}^{-3}$ depends entirely on the shape of the $n_{\mathrm{H}_{2}}$ profile. This profile is hollow, due to the high dissociation degree of the plasma and the short penetration depth of $\mathrm{H}_{2}$, limited by charge exchange with neutrals. We estimate the density of $\mathrm{H}_{2}$ to decrease significantly from a radius of $7 \mathrm{~mm}$ inwards and to be fairly well burnt out at a radius of $3-4 \mathrm{~mm}$. This is based on a charge exchange reaction rate of between $4 \times 10^{-15} \mathrm{~m}^{3} \mathrm{~s}^{-1}$ and $10^{-14} \mathrm{~m}^{3} \mathrm{~s}^{-1}$, reasonable estimations for the plasma conditions present. From the work of Vankan [32], we expect the molecular hydrogen in the beam to be highly rovibrationally excited. Krstić [27] shows that vibrational excitation can influence charge exchange reaction cross sections by a factor of 100 . We expect rotational excitation to increase the cross section even further. The charge exchange rate without rovibrational excitation is 4 $\times 10^{-17} \mathrm{~m}^{3} \mathrm{~s}^{-1}[26]$. Assuming a strong molecular density decay from a radius of $7 \mathrm{~mm}$ through $3-4 \mathrm{~mm}$, and emission being solely dependent on the molecular hydrogen density from a radius of $8 \mathrm{~mm}$ inwards, we can expect the strongest radiation at radii of around $7-8 \mathrm{~mm}$, corresponding to electron densities from Thomson scattering (Fig. 4) of $0.5-1.5$ $\times 10^{20} \mathrm{~m}^{-3}$. The expected emission profile is depicted schematically in Fig. 15.

\section{Discussion of two populations}

We now consider possible origins of the two (hot and cold) $\mathrm{H}^{*}(n=4)$ populations via their coupling with other species in the plasma. We show that the existence of two populations of radiating $\mathrm{H}^{*}(n=4)$ atoms is plausible.

The neutral atoms in the $n=4$ excitation state are produced by MAR as explained in Sec. IV A. The $\mathrm{H}_{2}{ }^{+}$ion resulting from the first MAR reaction (5) participates in an average of six ion-ion collisions before undergoing dissociative recombination. It thereby equilibrates with the ion temperature while also undergoing $\vec{E} \times \vec{B}$-drift rotation. In the next step, Eq. (6), the $\mathrm{H}_{2}^{+}$ion dissociatively recombines to form $\mathrm{H}^{*}$, which partly inherits the temperature and velocity 


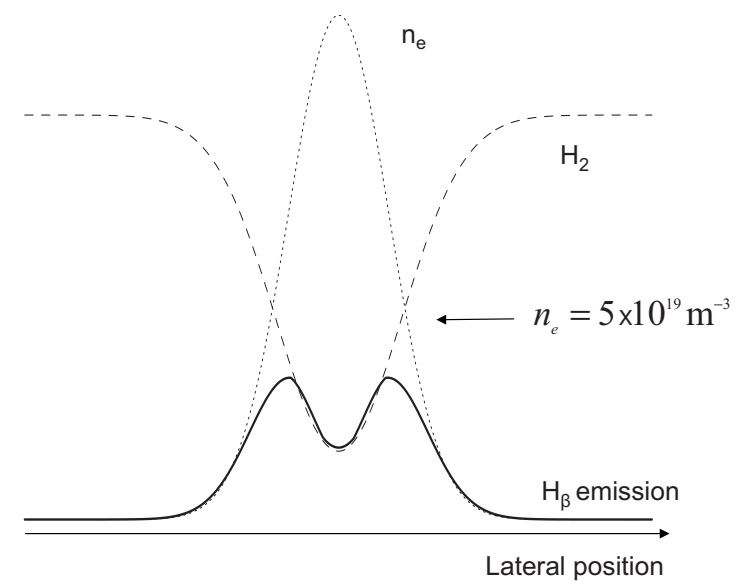

FIG. 15. Schematic picture of expected plasma emission on the basis of radial electron density profile and molecular hydrogen profile. The molecular hydrogen density profile is hollow due to a high dissociation degree and short penetration depth due to charge exchange. The emission is proportional to both electron density and molecular hydrogen density for low electron densities, however above about $n_{e}>5 \times 10^{19} \mathrm{~m}^{-3}$, the emission is solely proportional to $n_{\mathrm{H}_{2}}$. The result is that the measured emission originates mostly from a ring at a radius depending on the penetration depth of molecular hydrogen into the beam.

of the ions. A possible subsequent electron excitation to $n$ $=4$ does not change this. Next, the hot radiating species couple very effectively to the atomic ions by resonant charge exchange,

$$
\mathrm{H}^{+}+\mathrm{H}^{*} \rightarrow \mathrm{H}^{*}+\mathrm{H}^{+} .
$$

The time constant for charge exchange at an ion density of $1 \times 10^{20} \mathrm{~m}^{-3}$ is $4.6 \times 10^{-8} \mathrm{~s}$ [26]. This is shorter than the characteristic radiation time of the Balmer- $\beta$ transition, 1.2 $\times 10^{-7} \mathrm{~s}$. Thus, following the above consideration, we would expect the radiating $\mathrm{H}^{*}(n=4)$ atoms to have a velocity and temperature resembling those of the ion population.

However, particularly towards the edges of the plasma beam, where most emission originates, the radiating species also encounters neutrals: $\mathrm{H}$ atoms and $\mathrm{H}_{2}$ molecules. Collisions with $\mathrm{H}$ atoms are resonant and have large rates. With $n_{\mathrm{H}}$ in the same order as $n_{e}=n_{i}$, we estimate the time constant of these collisions (reaction cross section from [33]) to fall within the radiation time of the Balmer- $\beta$ transition. Thus part of the $\mathrm{H}^{*}(n=4)$ atoms will adopt a neutral, colder, and almost nonrotating distribution. As neutrals in the ground state have lower collision rates with ions (their mean-free path is of the same order as the diameter of the plasma beam) they will have clearly different properties to the ions. In conclusion, the Balmer- $\beta$ light will carry information both on the hot rotating ions and on the cold background gas.

These predictions are in line with results from hollow cathode arc plasmas in the literature where the ion motion can be measured. Theuws [10] experiments with argon plasma. A model predicts two neutral populations by considering on the one hand neutral-ion collisions and charge exchange reactions and on the other hand neutral-neutral collisions. The existence of these two populations is confirmed by

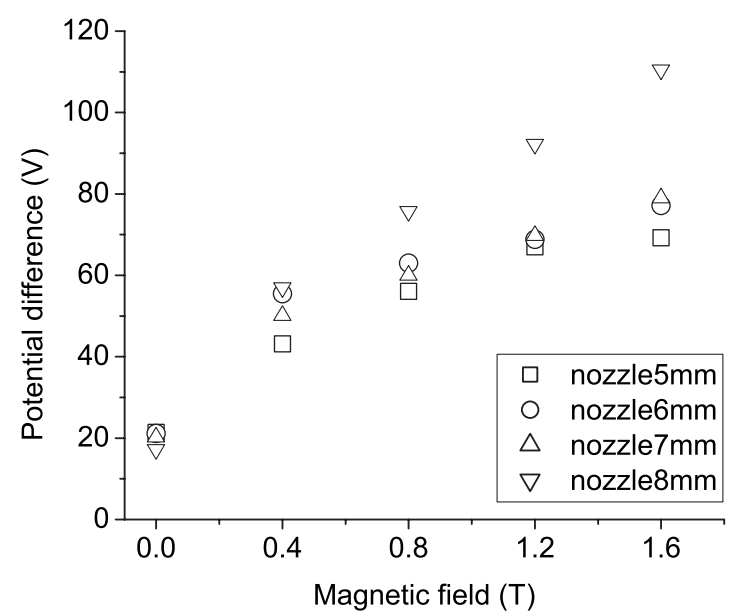

FIG. 16. Potential difference between the anode and the first cascaded plate plotted against the magnetic field for a nozzle diameter of 5, 6, 7, and $8 \mathrm{~mm}$. The voltage increases with both magnetic field and nozzle diameter.

a fit of a time-of-flight spectrum. Pots [9] also experiments in argon and fits a spectrum measured with a Fabry-Perot interferometer. He finds two populations which he explains as those neutrals that have undergone a charge exchange or neutral-ion collision and those that have not. Timmermans [7] finds similar results for argon with a Fabry-Perot interferometer.

\section{ROTATION OF THE PLASMA JET}

In this section we characterize the dependence of the jet rotation in Pilot-PSI on the magnetic field and nozzle diameter in order to explore the mechanisms behind the rotation. An understanding of these mechanisms is important since the electric fields associated with the rotation are responsible for higher power input into the plasma and this results in extra ionization and higher source efficiency.

Plasma rotation in a magnetized arc driven by an $\vec{E} \times \vec{B}$ force is well described in the literature (for example, [4-8]). In the absence of friction, inertia, and viscosity, the velocity of the $\vec{E} \times \vec{B}$ rotation drift is given by

$$
\vec{v}_{\text {rot }}=\frac{\vec{E} \times \vec{B}}{B^{2}} .
$$

In the Pilot-PSI plasma, two possible sources of the radial electric field are worth considering. They are the ambipolar field and the electric field that is related to the arc current. The ambipolar field in Pilot-PSI is a few hundred volts per meter, too small to account for the rotation velocities observed of kilometers per second.

The field causing the rotation is provided by the potential drop over the cascaded arc. In a magnetic field, the potential drop between the first plate of the cascaded arc and the anode (see Fig. 2) is larger than the potential drop between each of the other plates in the arc (see Fig. 3). The size of this extra potential drop is strongly dependent on magnetic field and the size of the anode nozzle as shown in Fig. 16. We can 


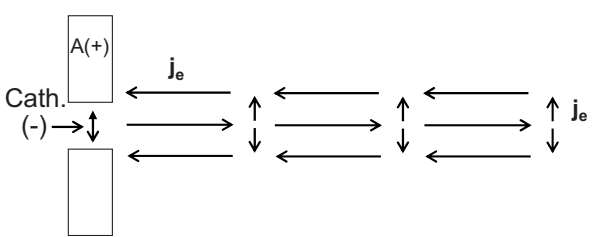

FIG. 17. The electrons cannot close the circuit within the source due to their limited mobility across the magnetic field. The radial electron current path spreads out in the axial direction until the resistance of the axial and radial current paths are equal.

explain the phenomena shown in Fig. 16 by considering the magnetic confinement of the beam. The resistivity is low in the axial direction parallel to the magnetic field and high in the radial direction perpendicular to the field. The radial electron current path therefore spreads out in the axial direction until the resistance of the axial and radial current paths are equal. This is shown schematically in Fig. 17. The resistance of this current channel causes a potential buildup between the first plate of the cascaded arc and the anode. The effect becomes stronger as the radial confinement increases with increasing magnetic field. The effect also increases with increasing nozzle opening radius, because the length traveled by the current in the vessel increases.

The radial electric field associated with the built-up potential can be estimated as the potential divided by the plasma radius. The field varies from 50 to 100 volts over the radius of a few millimeters and is much larger than the ambipolar field which corresponds to a voltage of around $1 \mathrm{~V}$ over the plasma radius. The $\vec{E} \times \vec{B}$-drift resulting from this field before inclusion of damping effects towards the edge of the beam is a strong rotation. Figure 18 shows such a strong rotation velocity, and its dependence on magnetic field and nozzle diameter. Plotted is the maximum velocity of the lateral profile. It increases with nozzle diameter and with magnetic field, in the same fashion as the potential buildup does in Fig. 16.

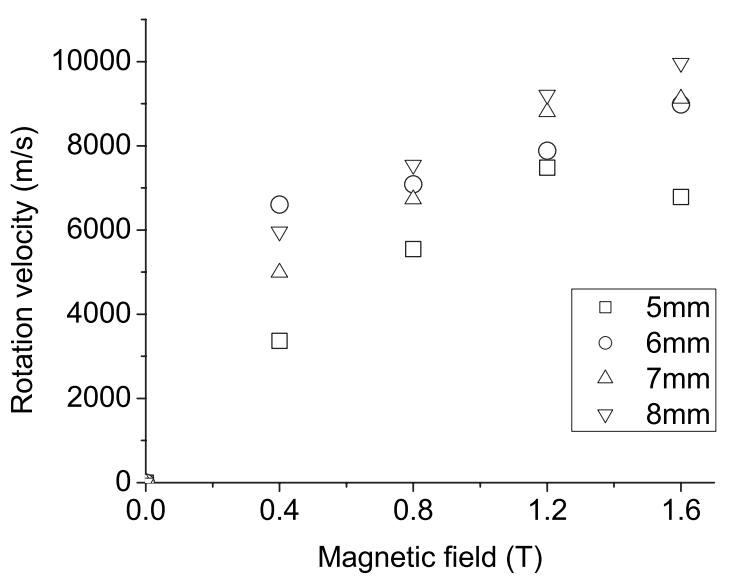

FIG. 18. Rotation velocities of the plasma jet (the maxima of the lateral profile) derived from the Doppler shift of the Balmer- $\beta$ line plotted against magnetic field. The measurements were performed for nozzle opening diameters of 5, 6, 7, and $8 \mathrm{~mm}$ for magnetic field settings, $0.4,0.8,1.2$, and $1.6 \mathrm{~T}$. The velocity increases approximately linearly with magnetic field.

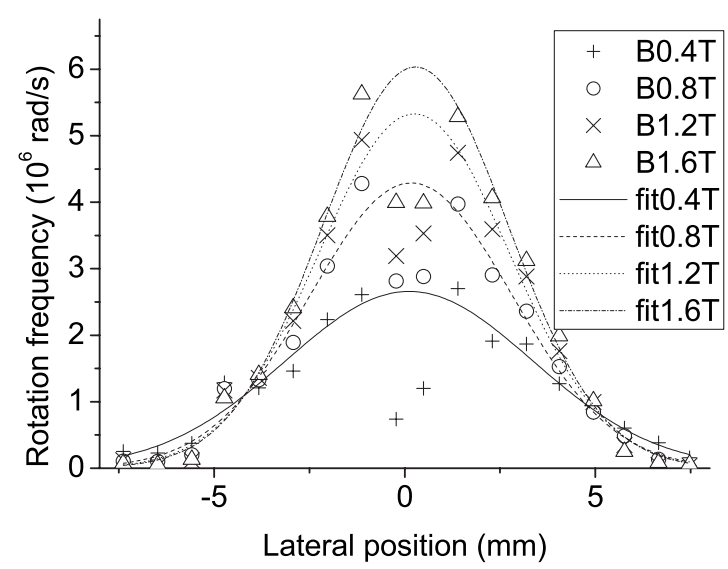

FIG. 19. The lateral rotation frequency profiles for each of the four magnetic field settings, $0.4,0.8,1.2$, and $1.6 \mathrm{~T}$.

To establish that the field strengths needed to drive the rotation are available, we compare the radial electric fields estimated from the potential build-up in the source to those needed to drive the rotation frequency. The measured rotation frequency is shown in Fig. 19 as a function of lateral position. The frequency is calculated as the rotation velocity in Fig. 10 divided by the lateral position at which it is measured. We take the central value from the fit of the rotation frequency and calculate the minimum electric field required to drive it, $E=v B=r \omega B$. We use the width of the rotation frequency profile in the calculation of the available electric field strength. A comparison of the available and minimum required electric field is shown in Fig. 20.

We observe that the electric fields available and required for rotation lie within the same range. For higher values of the magnetic field, the field available seems to be a little less than that required. This can be understood since we compare an average electric field to the field required for the maximum rotation frequency.

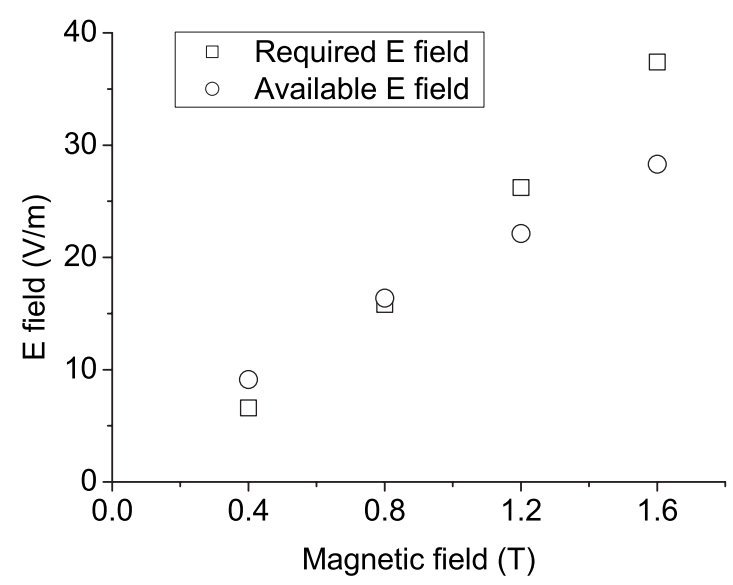

FIG. 20. A comparison between the electric fields available and those needed to drive the maximum rotation frequency. The available electric field is calculated from the potential drop between the anode and the first cascaded plate. The radial distance of this potential drop is taken to be the width of the rotation frequency profile. The maximum rotation frequency is taken to be the highest data point in the fit of the rotation frequency. The electric field required to drive it is calculated from $E=v B=r \omega B$. 


\section{CONCLUSION}

Large rotation velocities reaching $10 \mathrm{~km} / \mathrm{s}$ in the high density, magnetized plasma of Pilot-PSI have been measured. A correlation has been shown between the rotation velocity and both the magnetic field strength and the nozzle diameter of the cascaded arc plasma source. This supports the existence of an extra source of plasma heating, source current that penetrates axially into the vessel due to magnetic confinement. The potential buildup due to this current path has been shown to be strong enough to drive the rotation velocities measured by spectroscopy via an $\vec{E} \times \vec{B}$ force.

The rotation velocities observed support the methodology developed to analyze an asymmetric Balmer- $\beta$ optical emission spectrum in our plasma. The method involves fitting the spectrum with a double Voigt profile. This assumes the exis- tence of two radiating populations, whereby one is strongly coupled to the ion population. Results from the fitting procedure agree with predictions based on a survey of the reactions involved, the emission profile, and Thomson scattering measurements.

\section{ACKNOWLEDGMENTS}

This study is part of the research programme of the Stichting voor Fundamenteel Onderzoek der Materie (FOM), which is financially supported by the Nederlandse Organisatie voor Wetenschappelijk Onderzoek (NWO). It is supported by the European Communities under the contract of Association between EURATOM and FOM and carried out within the framework of the European Fusion Programme.
[1] G. J. van Rooij et al., Appl. Phys. Lett. 90, 121501 (2007).

[2] A. W. Kleyn, W. Koppers, and N. Lopes Cardozo, Vacuum 80, 1098 (2006).

[3] J. Westerhout et al., Phys. Scr., T T128, 18 (2007).

[4] V. M. Lelevkin, D. K. Otorbaev, and D. C. Schram, Physics of Non-equilibrium Plasmas (North-Holland, Amsterdam, 1992).

[5] D. C. Schram, I. J. A. M. v. d. Mullen, B. F. M. Pots, and C. J. Timmermans, Z. Naturforsch. A 38A, 289 (1983).

[6] H. Meyer, S. Klose, E. Pasch, and G. Fussmann, Phys. Rev. E 61, 4347 (2000).

[7] C. J. Timmermans, A. Lunk, and D. C. Schram, Contrib. Plasma Phys. 21, 117 (1981).

[8] F. Anderegg, R. A. Stern, F. Skiff, B. A. Hammel, M. Q. Tran, P. J. Paris, and P. Kohler, Phys. Rev. Lett. 57, 329 (1986).

[9] B. F. M. Pots, J. J. H. Coumans, H. J. Cornelissen, D. C. Schram, and B. van der Sijde, Proceedings of the 4th ESCAMPIG (C80), 132, 1978.

[10] P. G. A. Theuws, H. C. W. Beijerinck, D. C. Schram, and N. F. Verster, J. Appl. Phys. 48, 2261 (1977).

[11] O. Waldmann, H. Meyer, and G. Fussman, Contrib. Plasma Phys. 47, 691 (2007).

[12] C. M. van de Sanden, J. M. de Regt, G. M. Janssen, J. A. M. van der Mullen, D. C. Schram, and B. van der Sijde, Rev. Sci. Instrum. 63, 3369 (1992).

[13] G. M. W. Kroesen, D. C. Schram, and J. C. M. de Haas, Plasma Chem. Plasma Process. 10, 531 (1990).

[14] H. J. van der Meiden et al., Rev. Sci. Instrum. 79, 013505 (2008).

[15] T. J. Obernhuber, Phys. Chem. Chem. Phys. 5, 2799 (2003).

[16] C. Thomsen and V. Helbig, Spectrochim. Acta, Part B 46, 1215 (1991).

[17] J. M. M. J. Vogels, J. C. M. de Haas, D. C. Schram, and A.
Lunk, J. Appl. Phys. 59, 71 (1986).

[18] S. I. Braginskii, Reviews of Modern Physics, 1st ed. (Consultants Bureau, New York, 1965), Vol. 1, pp. 205-311.

[19] L. Spitzer, Physics of Fully Ionized Gases (Interscience, New York, 1962).

[20] V. Helbig and K.-P. Nick, J. Phys. B 14, 3573 (1981).

[21] C. G. Parigger, D. H. Plemmons, and E. Oks, Appl. Opt. 42, 5992 (2003).

[22] V. Helbig, Contrib. Plasma Phys. 31, 183 (1991).

[23] N. Ohno, N. Ezumi, S. Takamura, S. I. Krasheninnikov, and A. Yu. Pigarov, Phys. Rev. Lett. 81, 818 (1998).

[24] N. Ezumi, D. Nishijima, H. Kojima, N. Ohno, S. Takamura, S. I. Krasheninnikov, and A. Yu. Pigarov, J. Nucl. Mater. 266, 337 (1999).

[25] M. J. de Graaf, R. Severens, R. P. Dahiya, M. C. M. van de Sanden, and D. C. Schram, Phys. Rev. E 48, 2098 (1993).

[26] J. K. Janev, W. D. Langer, Jr., K. Evans, and D. E. Post, Elementary Processes in Hydrogen-Helium Plasmas (SpringerVerlag, New York, 1987).

[27] P. S. Krstić, Phys. Rev. A 66, 042717 (2002).

[28] R. P. Doerner, D. G. Whyte, and D. M. Goebel, J. Appl. Phys. 93, 5816 (2003).

[29] M. Y. Ye et al., J. Nucl. Mater. 313-316, 72 (2003).

[30] W. L. Wiese, M. W. Smith, and B. M. Glennon, Atomic Transition Probabilities (National Bureau of Standards, Washington, DC, 1966), Vol. 1.

[31] J. J. A. M. van der Mullen and D. C. Schram, Spectrochim. Acta, Part B 45, 233 (1990).

[32] P. Vankan, D. C. Schram, and R. Engeln, J. Chem. Phys. 121, 9876 (2004).

[33] T. Watanabe, Phys. Rev. 138, A1573 (1965). 